Virginia Commonwealth University VCU Scholars Compass

2014

\title{
Nanowire-based frequency-selective capacitive photodetector for resonant detection of infrared radiation at room temperature
}

Saumil Bandyopadhyay

Virginia Commonwealth University, sbandy@vcu.edu

Follow this and additional works at: http://scholarscompass.vcu.edu/egre_pubs

Part of the Electrical and Computer Engineering Commons

Bandyopadhyay, S. Nanowire-based frequency-selective capacitive photodetector for resonant detection of infrared radiation at room temperature. Journal of Applied Physics, 116, 023108 (2014). Copyright ( 2014 AIP Publishing LLC.

\section{Downloaded from}

http://scholarscompass.vcu.edu/egre_pubs/182

This Article is brought to you for free and open access by the Dept. of Electrical and Computer Engineering at VCU Scholars Compass. It has been accepted for inclusion in Electrical and Computer Engineering Publications by an authorized administrator of VCU Scholars Compass. For more information, please contact libcompass@vcu.edu. 


\title{
Nanowire-based frequency-selective capacitive photodetector for resonant detection of infrared radiation at room temperature
}

\author{
Saumil Bandyopadhyay ${ }^{\text {a) }}$ \\ Department of Electrical Engineering and Computer Science, Massachusetts Institute of Technology, \\ Cambridge, Massachusetts 02139, USA and Department of Electrical and Computer Engineering, \\ Virginia Commonwealth University, Richmond, Virginia 23284, USA
}

(Received 27 May 2014; accepted 26 June 2014; published online 11 July 2014)

\begin{abstract}
Characteristics of a capacitive infrared photodetector that works at room temperature by registering a change in capacitance upon illumination are reported. If used in an ideal resonant inductor-resistor-capacitor circuit, it can exhibit zero dark current, zero standby power dissipation, infinite detectivity, and infinite light-to-dark contrast ratio. It is also made frequency-selective by employing semiconductor nanowires that selectively absorb photons of energies close to the nanowire's bandgap. Based on measured parameters, the normalized detectivity is estimated to be $\sim 3 \times 10^{7}$ Jones for $1.6 \mu \mathrm{m}$ IR wavelength at room temperature. (C) 2014 AIP Publishing LLC.

[http://dx.doi.org/10.1063/1.4887515]
\end{abstract}

\section{INTRODUCTION}

A traditional photodetector senses light by undergoing a change in its electrical conductance under illumination. Its desirable properties are: (1) high detectivity to detect the faintest intensity of light; (2) low standby power dissipation for long battery life; (3) high light-to-dark contrast ratio (ratio of the conductance under illumination to that in the dark) to function well in a noisy environment; (4) frequency selectivity to lock on to particular frequencies of light at the exclusion of all other frequencies; (5) room temperature operation without the need for cryo-cooling; and (6) economy, ruggedness, robustness, and reliability.

Semiconductor based photodetectors find it difficult to meet most of these requirements. The challenges are especially severe for room temperature infrared (IR) detection since IR detectors are made of narrow bandgap semiconductors where phonons can excite electrons from the valence to the conduction band of the detector, causing a large current in the dark. Since the room temperature phonon population far exceeds the photon population (except in very high intensity radiation), the conductance of the photodetector under illumination $\left(G_{\text {light }}\right)$ will only slightly exceed that in the dark $\left(G_{\text {dark }}\right)$, so the light-to-dark contrast ratio $G_{\text {light }} / G_{\text {dark }}$ will barely exceed unity. The large dark conductance also leads to a large standby power dissipation $V_{0}^{2} G_{d a r k}$, where $V_{0}$ is the voltage of the battery powering the detector.

In the past, room-temperature IR detectors have been built from semiconductor structures fabricated with molecular beam epitaxy, ${ }^{1-6}$ but they are neither amenable to mass production nor sufficiently inexpensive for everyday use. There is one report of an inexpensive self assembled nanowire room-temperature IR detector that works in the inverse mode (its conductance decreases upon illumination). ${ }^{8}$ Recently, a tunneling IR detector and a wave functionengineered IR detector-both fashioned out of self-assembled

${ }^{\text {a)} E l e c t r o n i c ~ m a i l: ~ s a u m i l b @ m i t . e d u ~}$ nanowires electrodeposited in nanoporous anodic alumina films and both working in the normal mode-were demonstrated. ${ }^{9}$ They are cheap, rugged, and useful, with high roomtemperature detectivity (averaged over 3-5 $\mu \mathrm{m}$ wavelength), ${ }^{9}$ but their operation involves trap states, which are difficult to control and respond sluggishly to light. The light-to-dark conductance contrast ratio $G_{\text {light }} / G_{\text {dark }}$ is moderate $(<10: 1)$ and the dark conductance $G_{d a r k}$ is relatively large, resulting in significant standby power dissipation.

\section{CAPACITIVE PHOTODETECTOR}

In order to overcome these shortcomings, a novel capacitive photodetector was conceived and demonstrated. ${ }^{18}$ It conductance barely changes-but its capacitance changes measurably upon IR illumination. In other words, the conduction current flowing through the detector is virtually insensitive to light, but the displacement current is not. This has many advantages:

1. First, by using the capacitance in a resonant inductorcapacitor-resistor (LCR) circuit, even a very small change in capacitance upon illumination can be translated to a very high light-to-dark contrast ratio at room temperature. This ratio should be infinity if the inductor and capacitor are ideal and have no leakage resistance (i.e., the impedance of the capacitor is infinity at dc and the impedance of the inductor is infinity at infinite frequency).

2. Second, the "detectivity" of the detector will be very high at room temperature because of resonant detection. It should be infinity if the inductor and capacitor are ideal.

3. Third, the standby power dissipation should be negligible in the dark. It should be exactly zero if the inductor and capacitor are again ideal.

Such a capacitive photodetector can easily operate at room temperature. Moreover, if fashioned out of nanowires, it will be frequency-selective since semiconductor nanowires selectively absorb photons of energies at and slightly above their bandgap because of the quasi one-dimensional density 
of states. Finally, the nanowire capacitors discussed here are electrochemically self-assembled, which makes them massproducible and economical.

\section{A. Detection mechanism}

Capacitive photodetection is a relatively novel concept. It is very clear how light can change the conductance of a semiconductor by generating electron-hole pairs, but it is not so obvious how light can change the capacitance. The physical mechanism of how light can change capacitance is the following: ${ }^{18}$ The electron-hole pairs generated by light absorption in a semiconductor do not change the total amount of charge $\int d^{3} \vec{r} \rho(\vec{r}, t)$ since for every electron created there is a corresponding hole. However, the generated carriers can always change the instantaneous local charge density $\rho(\vec{r}, t)$ at an arbitrary location $\vec{r}$ in the semiconductor at time $t$. This will change the local instantaneous electric field $\overrightarrow{\mathcal{E}}(\vec{r}, t)$ in accordance with the Poisson equation $\vec{\nabla} \cdot \overrightarrow{\mathcal{E}}(\vec{r}, t)=\rho(\vec{r}, t) / \epsilon$, where $\epsilon$ is the detector material's permittivity. Since the displacement current density in a semiconductor under a time-varying bias is $\epsilon \partial \overrightarrow{\mathcal{E}}(\vec{r}, t) / \partial t$, it can change upon illumination. Note that the electric field $\overrightarrow{\mathcal{E}}(\vec{r}, t)$ has two contributions

$$
\overrightarrow{\mathcal{E}}(\vec{r}, t)=\overrightarrow{\mathcal{E}}_{a p p}(\vec{r}, t)+\overrightarrow{\mathcal{E}}_{s c}(\vec{r}, t),
$$

where $\overrightarrow{\mathcal{E}}_{a p p}(\vec{r}, t)$ is the electric field due to the applied (timevarying) voltage source $v(t)$, and $\overrightarrow{\mathcal{E}}_{s c}(\vec{r}, t)$ is the selfconsistent field caused by photogenerated electron-hole pairs. The displacement current flowing through the semiconductor under the time-varying voltage $v(t)$ is

$$
i_{\text {disp }}=C \frac{d v(t)}{d t}=A \epsilon \frac{\partial \overrightarrow{\mathcal{E}}(\vec{r}, t)}{\partial t},
$$

where $A$ is the cross-sectional area of the device and $C$ is the device capacitance. Since $\overrightarrow{\mathcal{E}}(\vec{r}, t)$ changes under illumination, but $v(t)$ does not, it is obvious that the capacitance will change under illumination. Clearly, depending on how the internal electric field is altered by light, the capacitance can either increase or decrease upon illumination.

Photocapacitance of solids is not unknown and has been observed before. ${ }^{10-12}$ An amorphous silicon sample that changes capacitance upon IR illumination was reported in Ref. 13. There, the capacitance increased upon illumination since absorbed IR photons excited electrons from extended to localized trap states in the bandgap of the amorphous semiconductor and changed the electric field distribution in the sample, leading to a change in capacitance. The photocapacitance however disappeared at signal frequencies exceeding $10 \mathrm{kHz}$ because the electric field inside the semiconductor could not change faster than it takes for the traps to charge or discharge, which may be several hundreds of microseconds. In order to observe photocapacitance at higher frequencies, the photogeneration process must be rapid and not involve traps, which fill or empty slowly. Valence-toconduction band photoexcitation of electrons in a semiconductor is a relatively fast process that takes perhaps a few nanoseconds in a direct-gap semiconductor. ${ }^{16}$ The resulting photogenerated electron-hole pairs can alter the electric field in the time scale of the "dielectric relaxation time" $\epsilon / \sigma(\sigma$ is the conductivity), which is fractions of a picosecond. Therefore, the response time of the photocapacitance will be a few nanoseconds (the radiative generation time of electron-hole pairs) as opposed to microseconds. The photocapacitance will therefore survive to much higher frequencies. High-frequency detection is superior to low-frequency detection since it is less vulnerable to $1 / f$ noise. A lower noise floor increases the detectivity of the photodetector.

It should be mentioned here that there are two previous reports of detecting IR radiation through heat-induced change in the capacitance of a structure upon IR illumination. The heat in the IR radiation was absorbed and caused a change in the capacitance. ${ }^{14,15}$ In those cases, heat energy was detected and not light energy, making those devices heat detectors rather than photodetectors. Since heating is a very slow process, the response times of such detectors would be very long. The capacitance change that occurred there is "thermocapacitance" rather than "photocapacitance".

\section{B. Resonant detection}

The photocapacitance (the relative change in capacitance upon illumination) is usually quite small in most materials, so the light-to-dark contrast ratio $i_{\text {disp }}^{\text {light }} / i_{\text {disp }}^{\text {dark }}$ $=C_{\text {light }} / C_{\text {dark }}$ will not be significantly different from unity. However, if one uses the detector as the capacitor in a resonant LCR parallel circuit as shown in Fig. 1 and excites it with a sinusoidal voltage whose frequency is the resonant frequency of the circuit in the dark, then the light-to-dark contrast ratio can be extremely large (ideally infinite) if the contrast ratio is defined as the ratio of the voltage across the series resistor under illumination to that in the dark

$$
\text { Contrast ratio }=\frac{\mathrm{V}_{\mathrm{R}}^{\text {light }}}{\mathrm{V}_{\mathrm{R}}^{\text {dark }}} \text {, }
$$

where $V_{R}$ is the voltage across the series resistor. This is true irrespective of how small the change in the capacitance is under illumination, as long as the capacitor and inductor are ideal and have no leakage.

\section{Ideal case}

Assume that the leakage resistance $R_{\text {leak }}$ shown in Fig. 1 is infinity, which is the ideal case. Also assume that the

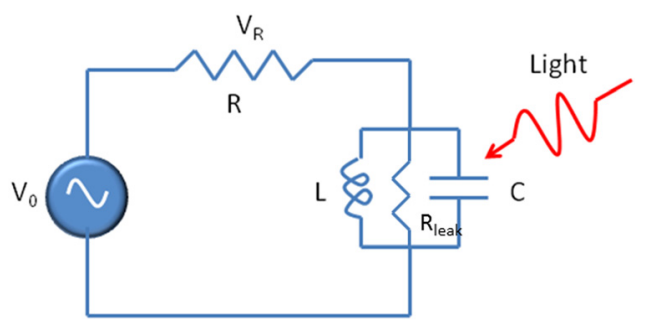

FIG. 1. A non-ideal resonant parallel inductor-capacitor circuit with a series resistor where the capacitor is light-sensitive. The parallel leakage resistance $R_{\text {leak }}$ is infinity if the inductor is perfectly insulating at infinite frequency and the capacitor is perfectly insulating at dc. 
detector's capacitance in the dark is $C_{\text {dark }}$, and under illumination is $C_{\text {light }}\left(C_{\text {dark }} \neq C_{\text {light }}\right.$ because of photocapacitance). The circuit is excited by a sinusoidal voltage of frequency $f_{0}=1 /\left(2 \pi \sqrt{L C_{\text {dark }}}\right)$. Therefore, the circuit is resonant in the dark, which makes the impedance of the parallel inductorcapacitor loop ideally infinity and the voltage drop over the series resistor $V_{R}^{\text {dark }}$ ideally zero. The conduction current $i_{\text {dark }}$ flowing through the resistor is then also ideally zero $\left(i_{\text {dark }}=V_{R}^{\text {dark }} / R\right)$, and the power dissipation $i_{\text {dark }}^{2} R$ is zero in the dark as well. That makes the standby energy dissipation exactly zero. Next, when light is turned on, the circuit goes out of resonance since the capacitance changes from $C_{\text {dark }}$ to $C_{\text {light }}$ and $f_{0}$ is no longer the resonant frequency $\left(f_{0}=1 /\right.$ $\left.\left(2 \pi \sqrt{L C_{\text {dark }}}\right) \neq 1 /\left(2 \pi \sqrt{L C_{\text {light }}}\right)\right)$. This makes the impedance of the inductor-capacitor loop finite, resulting in the flow of a conduction current $i_{\text {light }}$ through the resistor. Consequently, the voltage over the series resistor $\left(V_{R}^{\text {light }}=i_{\text {light }} R\right)$ becomes non-zero. As a result

Contrast ratio $=\frac{\mathrm{V}_{\mathrm{R}}^{\text {light }}}{\mathrm{V}_{\mathrm{R}}^{\text {dark }}}=\frac{\mathrm{i}_{\text {light }}}{\mathrm{i}_{\text {dark }}}=\frac{\mathrm{i}_{\text {light }}}{0}=\infty($ ideally $)$.

Thus, this device will ideally produce infinite light-to-dark contrast ratio and zero dark current or standby power dissipation, as long as the capacitance changes by a non-zero amount (no matter how small) upon illumination.

\section{Real case}

In reality, both the capacitor and the inductor will be "leaky," which will result in a parasitic leakage resistance $R_{\text {leak }}$ in parallel with the inductor and capacitor, as shown in Fig. 1. Taking this non-ideality into account, the light-todark contrast ratio will be

$$
\begin{aligned}
\frac{V_{R}^{\text {light }}}{V_{R}^{\text {dark }}}= & \frac{\left(R+R_{\text {leak }}\right) R_{\text {leak }}\left(1-\omega^{2} L C_{\text {light }}\right)+i \omega L\left(R+R_{\text {leak }}\right)}{R R_{\text {leak }}\left(1-\omega^{2} L C_{\text {light }}\right)+i \omega L\left(R+R_{\text {leak }}\right)} \\
\approx & \frac{R_{\text {leak }}\left(1-\omega^{2} L C_{\text {light }}\right)+i \omega L}{R\left(1-\omega^{2} L C_{\text {light }}\right)+i \omega L} \text { if } R_{\text {leak }} \gg R \\
= & \frac{R_{\text {leak }}\left(1-C_{\text {light }} / C_{\text {dark }}\right)+i \sqrt{L / C_{\text {dark }}}}{R\left(1-C_{\text {light }} / C_{\text {dark }}\right)+i \sqrt{L / C_{\text {dark }}}}
\end{aligned}
$$

where $\omega=2 \pi f_{0}=1 / \sqrt{L C_{\text {dark }}}$. Clearly, this ratio approaches infinity only if $R_{\text {leak }} \rightarrow \infty$.

The dark current in the circuit [assuming the applied voltage is $v(t)]$ is $v(t) /\left(R+R_{\text {leak }}\right)$, which approaches zero if $R_{\text {leak }} \rightarrow \infty$. The standby power dissipation in the circuit will be $V_{0}^{2} / 2\left(R+R_{\text {leak }}\right)$, where $V_{0}$ is the peak-to-zero amplitude of the sinusoidal voltage source $v(t)$. This quantity, too, approaches zero only if $R_{\text {leak }} \rightarrow \infty$.

\section{Frequency-selective photodetection}

If the capacitive photodetector is fashioned out of nanowires, then it can have one additional property. Bulk semiconductors absorb photons of all energies that exceed their bandgaps and hence detect light of all frequencies exceeding the threshold frequency $E_{g} / h\left(E_{g}=\right.$ bandgap, $h=$ Planck constant). However, in a semiconductor nanowire whose transverse dimensions are comparable to the DeBroglie wavelength of electrons and holes, the absorption spectrum becomes $^{16}$

$$
\alpha_{\text {nanowire }}(f) \propto \frac{1}{\sqrt{h f-E_{g}^{\prime}}}
$$

where $E_{g}^{\prime}$ is the blue-shifted bandgap in the nanowire (blueshift caused by quantum confinement). Therefore, only a narrow band of frequencies above $E_{g}^{\prime} / h$ is absorbed and detected, which makes nanowire photodetectors frequencyselective. ${ }^{9,17}$

\section{FABRICATION}

In order to fabricate the capacitive photodetectors, nanowires of GaSb were self assembled in anodic alumina pores of $50 \mathrm{~nm}$ diameter formed by anodization of alumina foils. ${ }^{18}$ The starting material is a $99.9999 \%$ pure aluminum foil that is diced into $2 \mathrm{~cm} \times 2 \mathrm{~cm}$ sized coupons. The latter were electropolished in a solution of perchloric acid, butyl cellosolve, ethanol, and distilled water at $40 \mathrm{~V}$ for $15 \mathrm{~s}$ (in installments of $5 \mathrm{~s}$ to reduce heating effects). The electropolishing reduced the root-mean-square surface roughness to $\sim 3 \mathrm{~nm} .{ }^{19}$ The electropolished coupons were then anodized in $0.3 \mathrm{M}$ oxalic acid under a dc voltage of $40 \mathrm{~V}$ using the aluminum sample as anode and platinum electrode as cathode. The anodization process resulted in the formation of an alumina film on the surface of the aluminum which contained a dense array of pores with nominal diameter $\sim 50 \mathrm{~nm} .^{20,21}$ The anodization lasted for 15 min and produced a $\sim 1.0 \mu \mathrm{m}$ thick alumina film. At the bottom of the pores, there was a $\sim 20 \mathrm{~nm}$ thick alumina "barrier layer" in contact with the aluminum substrate, which is a barrier to dc current flow along the pores but not to ac current flow. The pores were selectively filled with $\mathrm{GaSb}$ using ac electrodeposition carried out with a $4 \mathrm{~V}$ amplitude and $250 \mathrm{~Hz}$ frequency ac source. The electrolyte used for electrodeposition consisted of a mixture of $\mathrm{Ga}_{2}\left(\mathrm{SO}_{4}\right)_{3}, \mathrm{SbCl}_{3}$, and complexion agents such as trisodium citrate and citric acid dissolved in distilled water. GaSb selectively deposits within the pores since they offer the least impedance path for the ac current to flow. This resulted in the formation of a parallel array of 10-nm diameter GaSb nanowires in alumina. A cross-section scanning electron micrograph of a similar structure can be found in Ref. 17. The electrodeposition was continued until the surface just began to change color, signaling that the pores had been filled to the brim with slight overflow. Copper wires were then connected to the top surface with silver paste to complete the fabrication of photodetectors. The bottom aluminum substrate and the top contact served as the two plates of the capacitor.

A photograph of the sample is shown in Fig. 2(a), and the cross-section is depicted in Fig. 2(b).

\section{MEASUREMENT OF PHOTOSENSITIVE CAPACITANCE}

The capacitance of the sample was measured with an Agilent 4285A LCR meter in the signal frequency range of 


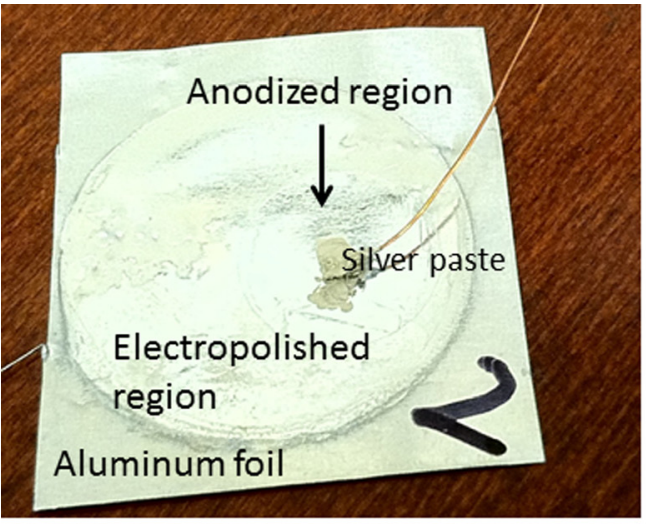

(a)

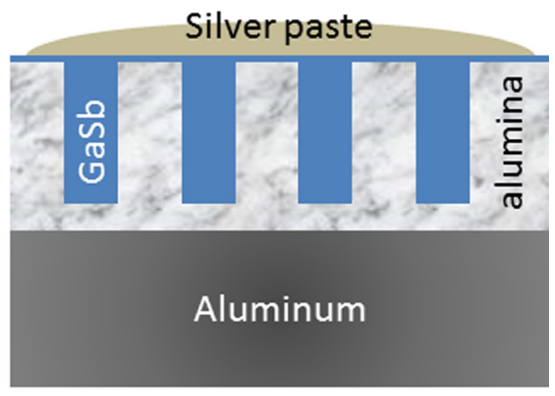

(b)

FIG. 2. (a) Photograph of a finished sample viewed from the top. (b) Schematic cross-sectional structure of the nanowire capacitors.

$75 \mathrm{kHz}-1 \mathrm{MHz}$ with no dc bias. This equipment did not allow measurement at any lower frequency. The capacitance was measured in the dark, under ambient room light, and under illumination by an IR lamp radiating in the wavelength range of $1-5 \mu \mathrm{m}$. The lamp was kept far enough away from the sample to avoid sample heating. The capacitance changed immediately upon illumination and thereafter did not drift with time, showing that there was no measurable heating effect on the capacitance. Fig. 3 shows the capacitance as a function of signal frequency under three conditions: dark, illuminated by room light, and illuminated by the IR lamp.

There are two important features to note in the data. First, the capacitance does change perceptibly upon illumination by the IR lamp, showing that there is measurable photocapacitance which is adequate for resonant photodetection.

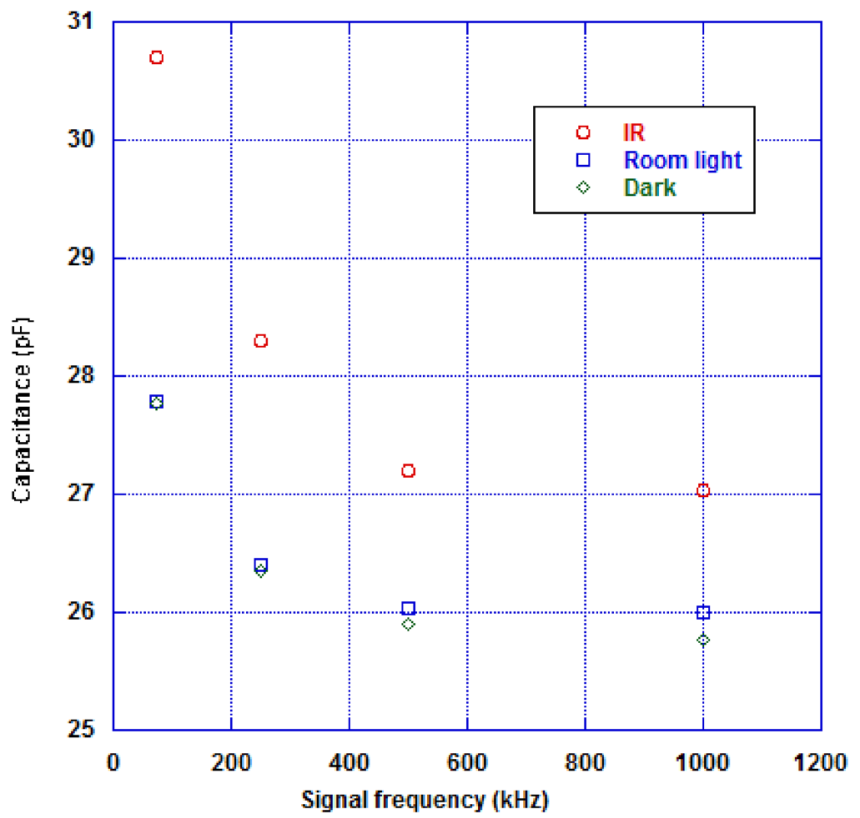

FIG. 3. Capacitance of the sample measured in the dark, under room light, and under IR illumination as a function of ac excitation frequency.
Note that we are measuring the capacitance of $\sim 10^{7}$ wires in parallel and the capacitances of some of these wires may increase while those of the others may decrease upon illumination since the change is determined by re-distribution of charge. Thus, there may be some cancellation effect, resulting in decreased photocapacitance; yet, the photocapacitance is very discernible. Second, the effect is clearly light frequencyselective. Room light (consisting of mostly visible light in the wavelength range of $0.4-1 \mu \mathrm{m}$ ) is not absorbed sufficiently and does not produce much change in the capacitance, but IR light (in the wavelength range $1-5 \mu \mathrm{m}$ ) is absorbed much more and causes a significant change in the capacitance. The bulk bandgap of GaSb is $720 \mathrm{meV}$ (Ref. 22) and quantum confinement in the nanowires increases it slightly. The increase was calculated using the prescription in Ref. 23 and was found to be only $1.16 \mathrm{meV}$, so the effective bandgap of the $50-\mathrm{nm}$ diameter $\mathrm{GaSb}$ nanowires is $771 \mathrm{meV}$. Therefore, the absorption spectrum of the nanowires should be peaked around $1.6 \mu \mathrm{m}$ wavelength. Since the IR lamp radiates in the range of $1-5 \mu \mathrm{m}$ wavelength, its light output is strongly absorbed and changes the capacitance noticeably by generating electron-hole pairs in the nanowires. Room light does not contain any appreciable $1.6 \mu \mathrm{m}$ wavelength component and is hence not sufficiently absorbed. That is why appreciable photocapacitance was measured under IR illumination, but not under room light illumination.

\section{RESONANT PHOTODETECTION}

Finally, a resistor-inductor-capacitor circuit was breadboarded with the nanowire sample as the capacitor. It was tested in the dark and under both room light and infrared illumination while driving it with a sinusoidal signal source. The resonant frequency $f_{0}$ was determined experimentally by tuning the frequency until the voltage over the series resistor became minimum in the dark. Since the capacitance was signal frequency dependent, the resonant frequency would have obeyed the non-linear equation

$$
f_{0}=\frac{1}{2 \pi \sqrt{L C\left(f_{0}\right)}} .
$$


The resonant frequency was experimentally found to be $45 \mathrm{kHz}$. The inductance of the inductor was $500 \mathrm{mH}$. Therefore, it was concluded that the capacitance of the sample was $\sim 100 \mathrm{pF}$ at the signal frequency of $45 \mathrm{kHz}$ (assuming that the inductance was relatively frequency-independent, which it should have been since it was a wire-wound inductor).

While driving the circuit with a $45 \mathrm{kHz}$ sinusoid of amplitude $10 \mathrm{~V}$, the voltage over the series resistor was monitored in the dark and under room light illumination in an analog oscilloscope. The results are shown in Fig. 4. Unfortunately, the oscilloscope was rather noisy, which did not allow a precise measurement of the light-to-dark contrast ratio, but this was a shortcoming of the measurement device and not the detector. No clear sinusoidal signal can be found in the trace when the sample is kept in the dark, but even if there is one, its amplitude is less than $2.5 \mathrm{mV}$. Under room light, there is a clearer hint of a sinusoidal signal with an amplitude of $3 \mathrm{mV}$. Under IR light, there is a much larger signal of amplitude $13.5 \mathrm{mV}$. Note, however, that the voltage over the series resistor is smaller under room light than under IR illumination. This is direct evidence of frequency-selective photodetection; the detector detects IR, but not room light.

\section{ESTIMATION OF VARIOUS QUANTITIES}

\section{A. The leakage resistance in the inductor-capacitor loop}

Since at resonance the combined impedance of the inductor-capacitor loop is $R_{\text {leak }}$, the leakage resistance $R_{\text {leak }}$ can be estimated from the relationship

$$
\frac{V_{R}^{\text {dark }}}{V_{0}}=\frac{R}{R+R_{\text {leak }}} .
$$

Since $V_{0}=10 \mathrm{~V}$ and $V_{R}^{\text {dark }} \leq 2.5 \mathrm{mV}$ (from the oscilloscope traces), one obtains $R_{\text {leak }} \geq 800 \mathrm{k} \Omega$.

\section{STANDBY POWER DISSIPATION}

From the above value of $R_{\text {leak }}$, one obtains that the standby power dissipation (in the dark) is $V_{0}^{2} / 2\left(R+R_{\text {leak }}\right)$ $=62.5 \mu \mathrm{W}$. This can be reduced by reducing $V_{0}$, but that will also reduce the detectivity (see later). In applications where a low standby power dissipation is more important than high detectivity, one will operate at a lower $V_{0}$.

\section{A. Responsivity}

The responsivity $\mathcal{R}$ is the change in the current through the circuit per unit incident infrared power. The IR lamp radiates $250 \mathrm{~W}$ within a solid angle of $\pi$. If it is placed at a distance $r$ from the sample surface, then the power incident per unit area on the sample is $250 \mathrm{~W} /\left(\pi r^{2}\right)$. In the experiments, $r=10 \mathrm{~cm}$. Therefore, the power incident on the photodetector is $250 \mathrm{~W} /\left(\pi r^{2}\right) \times 0.1 \mathrm{~cm}^{2}=79.5 \mathrm{~mW}$, since the area of the sample that is electrically probed (the so-called "effective area") is $\sim 0.1 \mathrm{~cm}^{2}$.

From the oscilloscope traces in Fig. 4, the amplitude of the voltage over the series resistor can be seen to change by $2.5 \times 5 \mathrm{mV}=12.5 \mathrm{mV}$ upon illumination. Since the
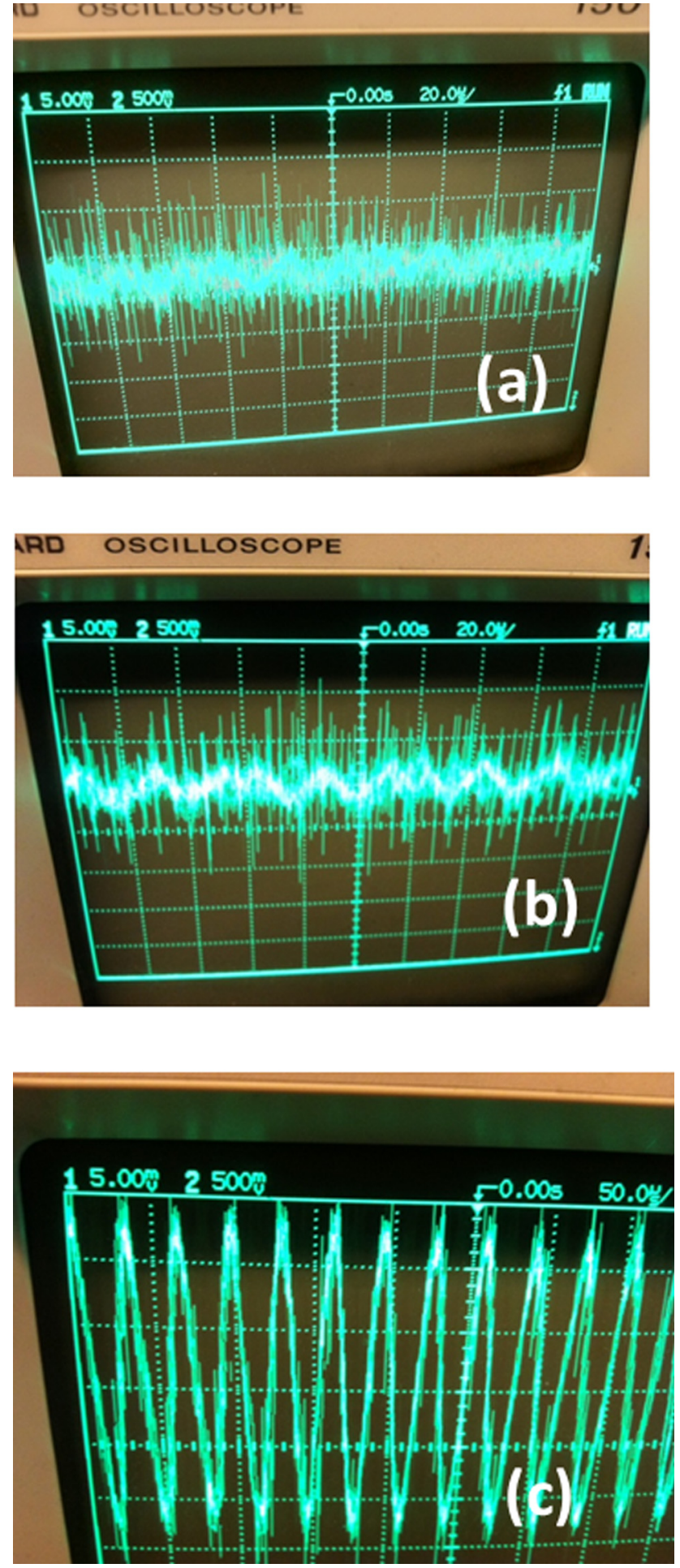

FIG. 4. Oscilloscope traces showing the voltage across the series resistor measured (a) in the dark, (b) under room light, and (c) under IR illumination when the circuit is driven by a sinusoid of peak-to-zero amplitude $10 \mathrm{~V}$. The series resistor was $200 \Omega$. The signal frequency was tuned in the dark until no sinusoidal voltage could be observed over the series resistor (above the noise floor), indicating that this is the resonant frequency in the absence of illumination. When light was turned on, a clear sinusoidal voltage was observed, well above the noise floor. An exact measurement of the contrast ratio $V_{\text {light }} / V_{\text {dark }}$ is not possible because of noise, but it is clearly quite large.

resistance of the series resistor is $200 \Omega$, the current in the circuit changes by $\Delta I=12.5 \mathrm{mV} / 200 \Omega=62.5 \mu \mathrm{A}$ when irradiated with $79.5 \mathrm{~mW}$ of IR power. Therefore, the responsivity $\mathcal{R}$ is $62.5 \mu \mathrm{A} / 79.5 \mathrm{~mW}=0.79 \mathrm{~mA} / \mathrm{W}$. 


\section{B. Minimum light-induced change in capacitance (photocapacitance) required for photodetection}

It is easy to show that when the LCR circuit is excited with a sinusoid of amplitude $V_{0}$ and frequency $f_{0}=\omega_{0} / 2 \pi$ $=1 / 2 \pi \sqrt{L C_{\text {dark }}}$, the amplitude of the current flowing through the series resistor in the dark is

$$
I_{\text {dark }}=\frac{V_{0}}{R+R_{\text {leak }}},
$$

and under illumination is

$$
I_{\text {light }}=\frac{V_{0}}{R+Z},
$$

where

$$
\begin{aligned}
Z & =\frac{i \omega_{0} L R_{\text {leak }}}{R_{\text {leak }}\left(1-\omega_{0}^{2} L C_{\text {light }}\right)+i \omega_{0} L} \\
& =\frac{i \sqrt{L / C_{\text {dark }}} R_{\text {leak }}}{R_{\text {leak }}\left(1-C_{\text {light }} / C_{\text {dark }}\right)+i \sqrt{L / C_{\text {dark }}}} .
\end{aligned}
$$

The signal current $I_{\text {sig }}$ is the difference between the current under illumination and in the dark

$$
\begin{aligned}
I_{\text {sig }} & =I_{\text {light }}-I_{\text {dark }}=V_{0}\left[\frac{1}{Z+R}-\frac{1}{R+R_{\text {leak }}}\right] \\
& =V_{0} \frac{1-\omega_{0}^{2} L C_{\text {light }}}{\left(1+\frac{R}{R_{\text {leak }}}\right)\left[R\left(1-\omega_{0}^{2} L C_{\text {light }}\right)+i \omega_{0} L\left(1+\frac{R}{R_{\text {leak }}}\right)\right]} \\
& =V_{0} \frac{1-\frac{C_{\text {light }}}{C_{\text {dark }}}}{\left(1+\frac{R}{R_{\text {leak }}}\right)\left[R\left(1-\frac{C_{\text {light }}}{C_{\text {dark }}}\right)+i \omega_{0} L\left(1+\frac{R}{R_{\text {leak }}}\right)\right]} \\
& =-V_{0} \frac{\Delta C}{\left(1+\frac{R}{R_{\text {leak }}}\right)\left[R(-\Delta C)+i \sqrt{L C_{\text {dark }}}\left(1+\frac{R}{R_{\text {leak }}}\right)\right]},
\end{aligned}
$$

where $\Delta C$ is the change in the capacitance induced by light $\left(\Delta C=C_{\text {light }}-C_{\text {dark }}\right)$.

The magnitude of the signal current is therefore

$$
\left|I_{\text {sig }}\right|=V_{0} \frac{|\Delta C|}{\left(1+\frac{R}{R_{\text {leak }}}\right) \sqrt{R^{2}(\Delta C)^{2}+L C_{\text {dark }}\left(1+\frac{R}{R_{\text {leak }}}\right)^{2}}} .
$$

The signal current's magnitude must exceed the total noise current in the resistor for detection. The noise current has a component due to thermal (Johnson-Nyquist) noise and another due to shot noise. Any $1 / f$ noise is neglected since the device operates at a frequency of several tens of $\mathrm{kHz}$. Therefore, the noise current is given by

$$
I_{\text {noise }}=\sqrt{4 k T G \Delta f / R}+\sqrt{2 q I_{\text {light }} \Delta f},
$$

where $k T$ is the thermal energy at temperature $T, q$ is the electron charge, and $\Delta f$ is the bandwidth of electrical detection. The first term is the thermal noise, and the second is the shot noise.

The condition for detection then becomes

$$
\left|I_{\text {sig }}\right| \geq I_{\text {noise }} .
$$

When operating at the edge of the noise limit, $\left|I_{\text {sig }}\right| \approx I_{\text {noise }}$, and hence the shot noise contribution to the noise current becomes negligible compared to the thermal noise contribution. Therefore, one gets from Eq. (14) that

$$
\lim _{\left|I_{\text {sig }}\right| \approx I_{\text {noise }}} I_{\text {noise }} \approx \sqrt{4 k T \Delta f / R} .
$$

Substituting Eqs. (13) and (16) into Eq. (15), one gets that the minimum photocapacitance needed for resonant photodetection in the presence of noise is

$$
\left|\Delta C_{\text {min }}\right|=\frac{\sqrt{4 k T L C_{\text {dark }} \Delta f / R}\left(1+\frac{R}{R_{\text {leak }}}\right)^{2}}{\sqrt{V_{0}^{2}-4 k T R \Delta f\left(1+\frac{R}{R_{\text {leak }}}\right)^{2}}} .
$$

For the values of $R, R_{\text {leak }}, L, C_{\text {dark }}$ (at the resonant frequency), and $V_{0}$ used in the experiments, $\Delta C_{\min }=6.4$ $\times 10^{-18} \mathrm{~F} / \sqrt{\mathrm{Hz}}$.

\section{Estimates of noise equivalent power and detectivity}

Assuming that the change in the current through the series resistor increases linearly with incident power (i.e., the responsivity is constant), the noise-equivalent-power (NEP) of the detector will be $\lim _{\left|I_{\text {sig }}\right| \approx I_{\text {noise }}} I_{\text {noise }} / \mathcal{R}$.

Using Eq. (16), with $R=200 \Omega$, one gets that $\lim _{\left|I_{\text {sig }}\right| \approx I_{\text {noise }}} I_{\text {noise }}=9 \mathrm{pA} / \sqrt{\mathrm{Hz}}$ at room temperature. Hence, $\mathrm{NEP}=11.6 \mathrm{nW} / \sqrt{\mathrm{Hz}}$ at room temperature. The normalized detectivity is defined as $D^{*}=\sqrt{A} / \mathrm{NEP}$, where $A$ is the cross-sectional area of the sample that is being electrically probed. Since this area is about $0.1 \mathrm{~cm}^{2}, D^{*}$ at room temperature is $2.7 \times 10^{7}$ Jones, which is comparable to the highest values reported in the literature for room-temperature IR detectors. ${ }^{2,4,17,24}$ References 6 and 7 reported a much higher responsivity, but they studied a single nanowire as opposed to $10^{7}$ nanowires and a single nanowire will always have higher responsivity because of the lack of ensemble averaging. However, a single nanowire does not produce much photocurrent because of its high intrinsic resistance. We

TABLE I. Measured and estimated parameters for the room-temperature nanowire-based capacitive infrared photodetector under an ac excitation voltage of $10 \mathrm{~V}$ amplitude and leakage resistance $>800 \mathrm{k} \Omega$.

\begin{tabular}{ll}
\hline \hline Standby power dissipation & $62.5 \mu \mathrm{W}$ \\
Responsivity & $0.79 \mathrm{~mA} / \mathrm{W}$ \\
Minimum photocapacitance for detection & $6.4 \mathrm{aF} / \sqrt{\mathrm{Hz}}$ \\
Noise equivalent power & $11.6 \mathrm{nW} / \sqrt{\mathrm{Hz}}$ \\
Detectivity & $2.7 \times 10^{7} \mathrm{Jones}$ \\
\hline \hline
\end{tabular}


could have of course increased the detectivity of our device by increasing the resistance of the series resistor.

All measured and estimated parameters (for an ac excitation voltage of $10 \mathrm{~V}$ amplitude and leakage resistance $>800 \mathrm{k} \Omega$ ) are summarized in Table I.

\section{CONCLUSION}

In conclusion, a nanowire capacitive photodetector is presented that resonantly detects infrared light at room temperature with high light-to-dark contrast ratio, low noise equivalent power, high detectivity, low dark current, and low standby power dissipation. The dark current can be made zero, while the detectivity, responsivity, and light-to-dark contrast ratio can be made infinity if the leakage resistance of the inductor/capacitor combination can be made infinite. Thus, there is no theoretical or fundamental obstacle to achieving excellent parameters, unlike in traditional IR photodetectors based on band-to-band photoexcitation of carriers, which can never achieve zero dark current at non-zero temperatures because of thermal excitation of carriers across the bandgap. A wide range of wavelengths can be detected by choosing different semiconductors that absorb different regions of the electromagnetic spectrum. Very long wavelengths can be detected if carriers are excited into the conduction band from shallow trap levels in the bandgap as opposed to the valence band. ${ }^{9}$ The detector reported here is cheap, rugged, robust, reproducible, and has a relatively long shelf-life (device works without noticeable degradation six months after fabrication, even though the device is not packaged or hermetically sealed). It requires an ac power source, but this is not a shortcoming since an on-chip oscillator can easily generate radio frequencies of few tens to few hundreds of $\mathrm{kHz}$ to act as the source.

\section{ACKNOWLEDGMENTS}

The author acknowledges past discussions with Dr. John Anderson and Dr. Supriyo Bandyopadhyay.
${ }^{1}$ A. Rakovska, V. Berger, X. Marcadet, B. Vinter, G. Glastre, T. Oksenhendler, and D. Kaplan, Appl. Phys. Lett. 77, 397 (2000).

${ }^{2}$ P. V. V. Jayaweera, S. G. Matsik, A. G. U. Perera, H. C. Liu, M. Buchanan, and Z. R. Wasilewski, Appl. Phys. Lett. 93, 021105 (2008).

${ }^{3}$ R. K. Singha, S. Manna, S. Das, A. Dhar, and S. K. Ray, Appl. Phys. Lett. 96, 233113 (2010).

${ }^{4}$ P. Bhattacharya, X. H. Su, S. Chakrabarti, G. Ariyawansa, and A. G. U. Perera, Appl. Phys. Lett. 86, 191106 (2005).

${ }^{5}$ Z. Liu, T. Luo, B. Liang, G. Chen, G. Yu, X. Xie, D. Chen, and G. Shen, Nano Res. 6, 775 (2013).

${ }^{6}$ J. Miao, W. Hu, N. Guo, Z. Lu, X. Zou, L. Liao, S. Shi, P. Chen, Z. Fan, T. C. Ho, T.-X. Li, X. S. Chen, and W. Lu, ACS Nano 8, 3628 (2014).

${ }^{7}$ L. Ma, W. Hu, Q. Zhang, P. Ren, X. Zhuang, H. Zhou, J. Xu, H. Li, Z. Shan, X. Wang, L. Liao, H. Q. Xu, and A. Pan, Nano Lett. 14, 694 (2014).

${ }^{8}$ N. Kouklin, L. Menon, A. Z. Wong, D. W. Thompson, J. A. Woollam, P. F. Williams, and S. Bandyopadhyay, Appl. Phys. Lett. 79, 4423 (2001).

${ }^{9}$ S. Bandyopadhyay, P. Agnihotri, and S. Bandyopadhyay, Physica E 44, 1478 (2012)

${ }^{10}$ R. Rosencher, N. Vodjdani, J. Nagle, P. Bois, E. Costard, and S. Delaitre, Appl. Phys. Lett. 55, 1853 (1989).

${ }^{11}$ J. Z. Zheng and J. W. Allen, Semicond. Sci. Technol. 5, 1013 (1990).

${ }^{12}$ N. B. Gorev, I. F. Kodzhespirova, E. N. Privalov, N. Khuchua, L. Khvedelidze, and M. S. Shur, Solid State Electron. 49, 343 (2005).

${ }^{13}$ D. Caputo, G. de Cesare, A. Nascetti, F. Palma, and M. Petri, Appl. Phys. Lett. 72, 1229 (1998)

${ }^{14}$ D. J. Sauer, R. U. Martinelli, R. Amantea, and P. A. Levine, U.S. patent 5,844,238 (1998)

${ }^{15}$ A. L. Micheli, J. V. Mantese, N. W. Schubring, and A. B. Catalan, U.S. patent 5,386,120 (1995).

${ }^{16}$ Supriyo Datta, Quantum Phenomenon, Modular Series in Solid State Devices (Addison-Wesley, Reading, MA, 1989).

${ }^{17}$ S. Bandyopadhyay, J. Mateo, and S. Bandyopadhyay, Physica E 43, 1255 (2011).

${ }^{18}$ S. Bandyopadhyay and J. Anderson, Appl. Phys. Lett. 102, 103108 (2013).

${ }^{19}$ R. E. Ricker, A. E. Miller, D.-F. Yue, G. Banerjee, and S. Bandyopadhyay, J. Electron. Mater. 25, 1585 (1996).

${ }^{20}$ N. Kouklin, L. Menon, and S. Bandyopadhyay, Appl. Phys. Lett. 80, 1649 (2002).

${ }^{21}$ N. Kouklin, S. Bandyopadhyay, S. Tereshin, A. Varfolomeev, and D. Zaretsky, Appl. Phys. Lett. 76, 460 (2000).

${ }^{22}$ S. M. Sze, Physics of Semiconductor Devices, 2nd ed. (Wiley-Interscience, New York, 1981).

${ }^{23}$ P. C. Sercel and K. J. Vahala, Phys. Rev. B 44, 5681 (1991).

${ }^{24}$ H. Lim, S. Tsao, W. Zhang, and M. Razeghi, Appl. Phys. Lett. 90, 131112 (2007). 